BULLETIN Bulletin hispanique

HISPANIQUE Université Michel de Montaigne Bordeaux

$115-2$ | 2013

Les traductions vieillissent-elles?

\title{
Lazarillo de Tormes "Lázaro de Tormes», edición, estudio y notas de Francisco Rico
}

Real Academia Española, Madrid, 2011

Jacques Joset

\section{(2) OpenEdition}

\section{Journals}

Édition électronique

URL : http://journals.openedition.org/bulletinhispanique/2942

DOI : 10.4000/bulletinhispanique.2942

ISSN : $1775-3821$

\section{Éditeur}

Presses universitaires de Bordeaux

Édition imprimée

Date de publication : 28 décembre 2013

Pagination : $773-777$

ISBN : 978-2-86781-908-7

ISSN : 0007-4640

\section{Référence électronique}

Jacques Joset, «Lazarillo de Tormes «Lázaro de Tormes», edición, estudio y notas de Francisco Rico », Bulletin hispanique [En ligne], 115-2 | 2013, mis en ligne le 14 février 2014, consulté le 22 septembre 2020. URL : http://journals.openedition.org/bulletinhispanique/2942 ; DOI : https://doi.org/10.4000/ bulletinhispanique.2942

Ce document a été généré automatiquement le 22 septembre 2020

Tous droits réservés 


\section{Lazarillo de Tormes «Lázaro de Tormes», edición, estudio y notas de Francisco Rico}

Real Academia Española, Madrid, 2011

Jacques Joset

\section{RÉFÉRENCE}

Lazarillo de Tormes «Lázaro de Tormes», edición, estudio y notas de Francisco RICo, Madrid, Real Academia Española, "Biblioteca clásica de la Real Academia Española, 29", 2011, XII + 322 pp.

1 Le 23 décembre 2000, Francisco Rico signait une pleine page du supplément littéraire «Babelia» (p. 14) du journal El País intitulée "...cómo no editar el Lazarillo». Cette charge doit laisser un souvenir que l'on imagine désagréable à l'auteur d'une édition commentée du prototype du roman picaresque parue cette même année 2000. Francisco Rico nous livrait en fait les recettes de " comment éditer le Lazarillo » : il lui suffisait de retourner en 2011 arguments, interprétations et méthode d'édition d'un livre dont on me dispensera de rappeler l'auteur et le titre.

2 Il est difficile, concédons-le, d'en remontrer à quelqu'un qui entretient avec le Lazarillo un dialogue de presqu'un demi-siècle. C'est en effet dès 1964 que le jeune Francisco Rico rédigeait les notes à sa première édition du Lazarillo, parue avec le Guzmán de Alfarache, dans le volume La novela picaresca española (Barcelona, Planeta, 1967). Vingt ans plus tard, la fameuse, mais pas toujours soignée, collection « Letras Hispánicas » de la maison Cátedra accueillait sa seconde édition réimprimée telle quelle "des douzaines de fois » (p. 216), même après la découverte en 1995 d'une nouvelle édition, celle de Medina del Campo, qui venait compléter (mais peut-être pas fermer) la série des premières éditions connues de 1554 (Alcalá de Henares A, Burgos B et Anvers C). Cette troisième et, augure-t-il, dernière édition tient évidemment compte de $\mathbf{M}$ pour 
l'établissement du texte critique. Cependant rappelons que le parcours "lazarillesque " de F. Rico est jalonné par des monographies ou recueils d'articles aussi essentiels que $L a$ novela picaresca y el punto de vista (1970) et Problemas del "Lazarillo» (1988). Cette troisième édition recueille donc les fruits de ces quelque dix lustres de tête à tête avec l'œuvre, résultats qui, comme toute pratique humaine, ne sont pas définitifs et restent perfectibles, mais gageons que les chercheurs qui mettraient en doute l'apport de Francisco Rico sur tel ou tel aspect de son œuvre critique en général admettront in petto que leur collègue de l'Université Autonome de Barcelone a fait faire des pas de géants aux études sur le Lazarillo de Tormes.

3 Ici on ne s'étonnera pas de retrouver des propositions déjà avancées dans l'un ou l'autre ouvrage ou éditions antérieurs comme ce « caso » qui est la clef structurale du récit. A la demande écrite d'un correspondant, identifié par la simple adresse courante de "Vuestra Merced", ami de l'Archiprêtre de San Salvador, Lazare répond par lettre en lui relatant el caso (avec article déterminatif) de long en large (muy por extenso) (p. 128). Le « cas » correspond à l'épisode des rumeurs qui courraient à Tolède à propos de la femme de Lazare et de l'Archiprêtre, qui, pour camoufler ses turpitudes, aurait imposé ce mariage au crieur public, fonction officielle mais infime à laquelle il avait accédé. Dédaignant les mauvaises langues, Lazarille peut légitimement se considérer arrivé " au sommet de toute bonne fortune ", au terme de son récit. Cette version du ménage à trois développée par Lázaro est une authentique prouesse littéraire en ce que l'auteur raconte l'histoire depuis sa naissance d'un personnage de basse extraction dont toute l'expérience (et celle de sa mère) explique son attitude de cocu consentant (p. 196).

4 Cette lecture du « cas », bien que connue depuis longtemps des lecteurs de F. Rico, sort revigorée et comme rajeunie dans son expression, tout comme celle du "réalisme " particulier de l'œuvre, énoncée dans son discours de réception à la Real Academia Española, qui héberge maintenant la collection de classiques espagnols dirigée par celui que l'institution accueillait en 1977. Le Lazarillo de Tormes est « réaliste » car il veut être appréhendé par le lecteur comme une lettre réellement écrite par un pregonero tolédan. Lazare signe donc un pacte de vraisemblance autobiographique avec le lecteur qui, en fait, lit le premier roman réaliste de la littérature occidentale (p.129), ce dont il prendra peut-être conscience à la fin de ce qu'il avait lu comme un exemple de plus d'un genre à la mode aux alentours de 1550, les carte messagiere ou lettere volgari (p. 146), intimement lié à la forme autobiographique. Ce détournement de la «lettre de nouvelles» au nom du vraisemblable est une carte maîtresse de l'art de l'auteur anonyme, aspect de la fiction souligné par F. Rico dès 1983 (pp. 144-145). Ce "réalisme» est à situer dans la perspective historique de la trajectoire du roman européen, comme forme (la missive à Sa Grâce), comme acte de langage et non comme matière (p. 155).

5 Ce « réalisme » est d'autant plus évident -voire choquant pour le lecteur de l'époque pris au piège par la lettre de Lazare- dans le panorama des lettres espagnoles entre 1545 et 1554 dont l' horizon élargi embrasse tant les œuvres originales que les rééditions d'œuvres remises à l'honneur et les traductions (pp.130ss). Arrivé à ce moment de mon propre dialogue avec le Lazarillo de Tormes (qui remonte à 1967), on me permettra d'insister sur l'influence exercée sur cette œuvre par L'âne d'or d'Apulée, admise par F. Rico (pp. 136, 172-173, 182, 185) et dont j'avais relevé l'importance dans la genèse de La lozana andaluza (1528) de Francisco Delicado via la traduction de López de 
Cortegana (1513) (voir mon "estudio preliminar" à l'édition de La lozana andaluza procurée par Folke Gernet et moi-même -Barcelona, Galaxia Gutenberg-Círculo de lectores, 2007, pp. LX-LXII-). J'avais aussi à cette occasion relevé des traces notables, mais cachées des dialogues de Lucien de Samosate dans l'œuvre de Delicado que nous retrouvons plus éparses dans le Lazarillo. Apulée, Lucien, Francisco Delicado et « Lázaro de Tormes ", voici un carré d'auteurs qu'il conviendra un jour de réexaminer en tant que tel. Ce carré se transforme en pentagone si l'on se souvient qu'un certain nombre de doses apuléennes et lucianesques se retrouvent également dans le Baldo (1542) (pp.138, 142), adaptation très libre du Baldus de Teófilo Folengo, dont l'importance dans la genèse du Lazarillo a été démontrée dans un article classique d'Alberto Blecua (1972-1973) repris, comme il se doit, dans la bibliographie.

6 Pour en terminer avec La lozana andaluza dans le Lazarillo, F. Rico réfute l'opinion répandue selon laquelle le personnage Lazarillo mentionné dans le mamotreto XXXV de l'œuvre de Delicado soit le même que le nôtre et qu'il était donc une figure folklorique connue. Selon l'éditeur-commentateur, le Lazarillo de La lozana andaluza renvoie en fait à une facétie répandue au $\mathrm{XV}^{\mathrm{e}}$ siècle, reprise dans Les cent nouvelles nouvelles. Il se sépare ainsi de Marcel Bataillon qui avait octroyé au folklore espagnol une place indue dans la genèse du Lazarillo (tout comme celui qui signe ces pages et qui, dans son péché de jeunesse de 1967, avait peut-être aussi surestimé la prolongation dans notre roman de traditions médiévales, encore que...). Or l'étude de F. Rico est dédicacée "à la mémoire de Marcel Bataillon» (p.90). Cette dédicace doit être comprise comme un aveu de filiation intellectuelle aussi exigeante en matière de philologie qu'en histoires de la littérature et de la pensée espagnoles. C'est dire que la réduction du substrat folklorique du Lazarillo ne va pas sans regrets sincères de sa part ("Duele disentir de un maestro como Bataillon, [...] », p. 156), largement atténués par nombre de renvois positifs au maître français dont, par exemple, l'approbation de l'interprétation que celui-ci donna à l'expression «crear de nuevo [el mundo]» (p. 43) comme expression triviale de la fabrication à partir de rien et le rejet de celle d'Américo Castro, qui y avait vu un trait stylistique hispano-hébreu sorti de la plume d'un auteur converso (p. 270, sub 43.1). Une telle rencontre n'est pas à dédaigner dans le contexte toujours brûlant de la recherche de paternité du Lazarillo, en particulier de son identification à un « converti ».

7 A ce sujet, on sait que, depuis toujours, F. Rico a revendiqué l'anonymat comme statut consubstantiel au Lazarillo. S'agissant d'une carta mensajera d'un crieur public de Tolède, l'auteur se devait de cacher soigneusement son identité. L'œuvre est un vrai " apocryphe ", attribué à un faux auteur (p.116). Cette position, F. Rico l'inscrit jusque dans la page de titre de cette troisième édition, qui, à la ligne dévolue à l'auteur, pose un "Lázaro de Tormes ", ainsi, entre guillemets.

8 Ceci ne l'empêche nullement de passer en revue les divers candidats à la paternité du roman (pp. 118ss.) depuis le hiéronymite Juan de Ortega jusqu'à Alfonso de Valdés (sur une échelle de probabilités allant du moins au plus invraisemblable) en passant par la personnalité la plus revendiquée historiquement mais la plus trompeuse littérairement, Diego Hurtado de Mendoza, et Sebastián de Horozco. Aucun n'échappe au crible serré de la critique de notre éditeur.

9 Quoi qu'il en soit du nom propre qui s'occulte derrière "Lázaro de Tormes », celui-ci rédigea son œuvre, sans le titre que nous lui connaissons (La vida de Lazarillo de Tormes, $y$ de sus fortunas y adversidades) ni la division en tractados (pp. 96-98) ${ }^{1}$ entre novembre 1551 
et la princeps - peut-être précédée d'une courte circulation manuscrite, comme une lettre authentique. Aucun exemplaire de cette première édition, datable au plus tôt de la seconde moitié de 1552 et au plus tard de fin 1553, ne nous est parvenu, mais son format doit être un in octavo comme A, B et $\mathbf{M}$ et son aspect matériel proche du petit volume de Medina del Campo (p. 100). Le terminus a quo de la rédaction nous est fourni par la pragmatique sur les taux de change (p. 110) à laquelle ferait référence une bourle au détriment de l'aveugle (ya iba de mi cambio aniquilada en la mitad del justo precio, p. 16) dont la base légale aurait été décelable par les contemporains: Le terminus ad quem proche des quatre premières éditions actuellement connues a été avancé par F. Rico dès 1987. On le voit, les deux événements historiques mentionnés dans l'œuvre, sujets de discussions entre érudits, la bataille de los Gelves (Djerba), sans aucun doute celle de 1510, et les Cortes de Tolède accompagnées d'une entrée triomphale de l'Empereur dans la ville, plus que plausiblement celles de 1538-1539, ne servent que fort peu à la datation du Lazarillo (pp.102-106). C'est que "Lázaro de Tormes " ne prétend pas à l'exactitude historique : il lui suffit de situer l'action dans un passé récent dont le protagoniste évoque des événements connus de tous (p. 106). L'important pour lui est de garantir l'historicité du protagoniste-narrateur qui force le lecteur à le donner pour véridique. Tout commentaire insuffisamment ou pas du tout documenté historiquement fait l'objet, sur ce point comme sur tous ceux qui configurent l' «Estudio » de F. Rico, d'un rejet impitoyable, la plupart du temps en notes de bas de page, marque supplémentaire de distanciation parfois marquée au coin de l'ironie (voir pp. 99 , n. $12 ; 105$, n. $8 ; 112 ;$ n. $25 ; 159$, n. $14 ; 204$, n. 19).

tes contextes socio-historiques et des matériaux issus des traditions littéraires et folkloriques, quitte à revoir à la baisse la cote de ces derniers, intégrés à l'encyclopédie de tout lecteur des environs de 1550 pour que celui de notre temps évalue le prix de l'élaboration d'un vraisemblable romanesque nouveau (pp. 159-169).

11 Le texte critique est basé sur la collation des quatre éditions conservées de 1554 et, le cas échéant, des conjectures du Lazarillo castigado de Juan López de Velasco de 1573 (pp. 197 et 203). Vigoureux défenseur de la «bibliographie textuelle » et contempteur de la théorie lachmanienne en matière d'ecdotique des textes imprimés du Siècle d'or, basée, comme on le sait, sur les fautes communes et la construction d'un stemma, F. Rico applique au Lazarillo (pp 197-198) les connaissances acquises sur les méthodes de travail des ateliers typographiques des $\mathrm{XVI}^{\mathrm{e}}$ et XVII ${ }^{\mathrm{e}}$ siècles longuement exposées dans son $\mathrm{El}$ texto del "Quijote » (Barcelona, Centro para la Edición de los Clásicos Españoles-Destino, 2005). Cependant la seule ratio typographica n'est pas toujours applicable et la reconstruction de la princeps, avoue l'éditeur, frise l'impossibilité. Le texte proposé par celui-ci est donc éclectique en ce qu'il résout les difficultés des leçons divergentes au cas par cas.

12 Les notes de bas de page, qui visent au déchiffrement littéral lexico-grammatical indispensable au lecteur du XXI siècle, sont abondantes et toujours judicieuses. Elles renvoient souvent à des notes complémentaires, destinées aux lecteurs curieux et désireux d'en connaître plus. L'apparat critique, quant à lui, s'adresse aux philologues purs et durs. Ces notes, prises ensemble, constituent en elles-mêmes un commentaire et une herméneutique du Lazarillo dont on ne pourra désormais se dispenser. On n'en sautera donc aucune depuis la première consacrée au titre de l'œuvre (p. 2) avec sa complémentaire (p. 235) jusqu'à la dernière (p.80, n.5) et sa complémentaire 
(pp. 295-297) sur l'entrée triomphale de Charles-Quint et les Cortes de Tolède. L'ensemble est parcouru par un tissu serré de références à des œuvres de l'Antiquité classique, directes ou parvenues aux alentours de 1550 par des intermédiaires surtout $\mathrm{du} \mathrm{XV}$ siècle, et un substrat biblique. Celles-là et celui-ci caractérisent un « Lázaro de Tormes" humaniste, également attentif aux realia: une note entre mille de cette dernière catégorie est consacrée à l'usage réel de la bouche comme porte-monnaie à l'époque (p. 40, n. 5).

Quant à la présentation générale et les problèmes graphiques suscités par l'édition de toute œuvre ancienne, ils sont tranchés selon les critères généraux de la collection de la «Biblioteca clásica», dès sa première version de "Círculo de lectores-Galaxia Gutenberg " (pp. 212-213). Ils obéissent à la seule volonté d'accessibilité du texte au lecteur du XXI siècle, d'où l'option de choisir la variante graphique moderne qui figure dans une ou plusieurs éditions de 1554. La modernisation bien mesurée, valable aussi pour la ponctuation, est le seul guide de notre éditeur qui, pour donner un exemple, choisira systématiquement Arcipreste en écartant Acipreste.

Toute entreprise humaine est perfectible, disais-je au début de cette recension, celle-ci comme une autre. Et cette troisième et dernière (???) édition du Lazarillo de Tormes de F. Rico gagnerait encore dans sa forme en éliminant dans de futurs retirages (qui, je le gage, ne manqueront pas) des coquilles telles que incursas pour incursan (p. 105, 1.9), pág. 169 pour pág. 161 (p.134, n. 15, 1.3), Mystère des Artes des Apotres pour ...Actes... (p. 161, 1. 20), L'enfant pour L'enfant (p. 171, n. 35, 1. 1, -apostrophe inversée-), problémes pour problèmes (p. 175, n. 47, 1. 2), p asa pour pasa (p. 195, 1. 22), Amberes y Amberes pour Alcalá y Amberes (p. 213, n. 9, dernière ligne), riñiendo riñiendo pour riñendo riñiendo (p. 214, 1. 16). On devra réinsérer aussi dans la bibliographie, par ailleurs très complète si non exhaustive (mais doit-elle l'être ?), des références données sous forme abrégée dans le texte ou les notes comme B. Geremek [1991] (p. 135, n. 18, 1.2), J. Fradejas Lebrero [1984] (p. 193, n. 97, dernière ligne), B. Brancaforte 1982 (p. 211, 1. 15), C. R. Rabell 1996 (p.211, 1.20). Manque également une note complémentaire à $\mathbf{5 1 . 6}$ annoncée en note de bas de page (p. 51).

Mais tout cela n'est que peccata minuta facilement remédiable et de toute façon bien pardonnable au regard du trésor d'intelligence, de rigueur philologique, d'exigence historique et d'élégance stylistique déployé par Francisco Rico dans cette édition qui deviendra une référence obligatoire pour tout chercheur sur l'œuvre de «Lázaro de Tormes » ou, plus simplement pour tout amateur de bonne littérature...en croisant les doigts pour que cette race ne disparaisse pas en ce $\mathrm{XXI}^{\mathrm{e}}$ siècle qui en est toujours à une aube brumeuse. Le Lazarillo mérite mieux que de rester la proie d'une chapelle de spécialistes. Autant offrir aux uns et aux autres la possibilité de le lire dans la meilleure édition actuellement sur le marché. 


\section{NOTES}

1. Cette suppression de la division en "tractados" et la prise en compte de l'édition de Medina del Campo figuraient déjà dans des éditions plus " occasionnelles » de 1999-2000 (énumérées p. 216, n. 17) publiées avec la collaboration de Fernando Cabo Aseguinolaza.

\section{AUTEURS}

JACQUES JOSET

Université de Liège 